cardiomyopathy and suggests exciting new targets for BH4based therapeutics.

\section{HUMAN OXIDISED PHOSPHOLIPID MACROPHAGES HAVE HIGH LIPOPROTEIN HANDLING CAPABILITIES WITHOUT READILY FORMING UNWANTED FOAM CELLS}

${ }^{1}$ Kajus Baidzajevas*, ${ }^{2}$ Eva Hadadi, ${ }^{3}$ Bernett Lee, ${ }^{3}$ Josephine Lum, ${ }^{4}$ lan Sudbery, ${ }^{3}$ Sarah Lai, ${ }^{3}$ Siew Cheng Wong, 'Endre Kiss-Toth, ${ }^{1}$ Heather Wilson. 'Department of Infection, Immunity and Cardiovascular Disease, Medical School, University of Sheffield, S10 2RX, UK; ${ }^{2}$ Laboratoire ESTeam UMRS 935-Batiment A, 7 rue Guy Moquet, F-94800 Villejuif, France; ${ }^{3}$ Singapore Immunology Network (SIgN), A *STAR, Immunos Building, 8A Biomedical Grove, 138648, Singapore; ${ }^{4}$ Department of Molecular Biology and Biotechnology, University of Sheffield, S10 2TN, UK

\subsection{6/heartjnl-2017-311726.199}

Rationale Cardiovascular disease is the leading cause of death world-wide. Macrophages are crucial in regulating the plaque environment, especially the lipid content. However, current characterisation of macrophage phenotypes lipoprotein handling capacity is conflicting and incomplete. We hypothesised that lipoprotein handling differed among distinct macrophage phenotypes due to differential gene and protein expression. We tested this using a range of functional, gene and protein expression assays.

Methodology Monocytes were isolated from healthy donor blood by gradient centrifugation and magnetic selection, then differentiated into macrophages over 7 days using M-CSF. Macrophages were polarised over 24 hour by IFN $\gamma+L P S$, IL4, IL-10, oxPAPC (oxidised phospholipid) and CXCL4, respectively. Unpolarised macrophages were used as controls.

Foam cell formation was determined by Oil-Red-O staining and acLDL uptake was detected by flow cytometry. Cholesterol content and efflux were measured using colorimetric and fluorescent assays. RNA expression was determined by RNAseq and qRT-PCR and cell surface protein expression was measured by flow cytometry.

Results IFNÃ $\square \hat{A}^{3}+$ LPS macrophages did not readily form foam cells (0.23 compared to unpolarised) or process lipoprotein particles, whereas IL-4 and IL-10 polarised macrophages displayed the highest capacity in foam cell formation (1.02 and 1.08 compared to unpolarised) and lipoprotein handling. OxPAPC macrophages exhibited lipoprotein processing capabilities similar to IL-4 and IL-10 macrophages, but did not readily form foam cells (0.22 compared to unpolarised). CXCL4 macrophages displayed intermediate foam cell formation (0.84 compared to unpolarised) and lipoprotein handling capabilities.

Differences in foam cell formation and lipoprotein uptake correlated directly to specific scavenger receptor gene and protein expression. Only IFN $\gamma+$ LPS macrophages had significantly reduced expression of key internal lipoprotein processing genes (q 0.0001). Cholesterol efflux correlated directly to specific $\mathrm{ABC}$ transporter protein, but not RNA expression.

Conclusions. In vitro human macrophage phenotypes differ in foam cell formation and lipoprotein handling capabilities that are associated with differential key gene and protein expression.

\section{THERAPEUTIC RESOLUTION OF PULMONARY ARTERIAL HYPERTENSION (PAH) BY NOVEL SMALL MOLECULE NATURAL PRODUCTS}

$1,2 \mathrm{~N}$ Sharmin* ${ }^{3} \mathrm{H}$ Malik, ${ }^{3} \mathrm{~A}$ Siddiqui, ${ }^{3} \mathrm{H}$ Chowdhury ${ }^{3} \mathrm{~S}$ Kanagenti, ${ }^{4} \mathrm{~A}$ Graham, ${ }^{1} \mathrm{C}$ Wright, ${ }^{5} \mathrm{~N}$ Morrell, ${ }^{3} \mathrm{RC}$ Trembath, ${ }^{1,3,6,7} \mathrm{MT}$ Nasim* ${ }^{*}{ }^{1}$ School of Pharmacy, University of Bradford; ${ }^{2}$ Dept. of Pharmaceutical Technology, University of Dhaka, Bangladesh; ${ }^{3}$ Dept. of Medical and Molecular Genetics, King's College London; ${ }^{4}$ School of Medical Sciences, University of Bradford; ${ }^{5}$ Dept. of Medicine, University of Cambridge; ${ }^{6}$ NIHR Biomedical Research Centre, King's College London, UK; ${ }^{7}$ Centre for Health, Agriculture and Socio-Economic Advancements (CHASA), Lalmonirhat, Bangladesh

\subsection{6/heartjnl-2017-311726.200}

Heterozygous germline mutations in the gene encoding type II bone morphogenetic protein receptor (BMPRII) underlie the majority $(\sim 80 \%)$ of the familial form of pulmonary arterial hypertension (HPAH). ${ }^{1}$ PAH is a devastating cardiovascular disorder caused by narrowing of blood vessels in the lungs. We earlier demonstrated that mutations in BMPR2 impinge upon the BMP signalling pathway and potentiate the TGF- $\beta^{2}$ signalling leading to abnormal proliferation and apoptosis resistance of endothelial and pulmonary arterial smooth muscle cells (PASMCs). ${ }^{2,3}$ No cure for this disorder is known. Traditional therapies aim to improve cardiopulmonary function and were established before recognising the involvement of substantial genetic components of PAH. Hence, there is an urgent need to identify novel compounds capable of providing therapeutic intervention prior to or following the onset of disease.

High-throughput cell based BMP-responsive screens were carried out, which identified several natural compounds as hits. The hit compounds were optimised through a medicinal chemistry programme. These compounds were tested in a number of cell based experiments including reporter assays, gene expression studies and western blot analyses. The compounds CRT-01, CRT-02 and CRT-03 enhanced BMP signalling in BMP responsive reporter assays. Moreover, these compounds were able to induce BMP responsive id1 gene expression and increased the phosphorylation of SMAD1/5 proteins. Furthermore, these compounds inhibited excessive proliferation of PASMCs harbouring a pathogenic BMPR2 mutation. Taken together, this study identified novel compounds eliciting pro-BMP effects which may have experimental and clinical applications in $\mathrm{PAH}$.

\section{REFERENCES}

1. Lane KB, Machado RD, Pauciulo MW, Thomson JR, Phillips JA, Loyd JE, Nichols WC, Trembath RC. Heterozygous germline mutations in BMPR2, encoding a TGF$\hat{i}^{2}$ receptor, cause familial primary pulmonary hypertension. Nature genetics 2000;26(1):81-84

2. Nasim MT. Ghouri A, Patel B, James V, Rudarakanchana N, Morrell NW, Trembath RC. Stoichiometric imbalance in the receptor complex contributes to dysfunctional BMPR-II mediated signalling in pulmonary arterial hypertension. Hum Mol Genet 2008;17(11);1683-94.

3. Nasim MT, Ogo T, Chowdhury HM, Zhao L, Chen CN, Rhodes C, Trembath RC BMPR-II deficiency elicits pro-proliferative and anti-apoptotic responses through the activation of TGFbeta-TAK1-MAPK pathways in PAH. Hum Mol Genet 2012;21(11):2548-58. 